\title{
SÍNDROME DE BURNOUT NA EQUIPE DE ENFERMAGEM DE UM HOSPITAL UNIVERSITÁRIO
}

Renata Cristina Gasparino ${ }^{1}$

Enfermeira. Mestre em Enfermagem. Universidade de Campinas. Jundiaí-SP-Brasil

RESUMO: O objetivo deste estudo foi identificar o nível da Síndrome de Burnout da equipe de enfermagem e sua relação com as variáveis sobrecarga de trabalho, satisfação profissional e intenção em deixar o emprego. A coleta de dados foi realizada entre junho e agosto de 2011 utilizando-se o Inventário de Burnout de Maslach. A amostra de 178 profissionais demonstrou moderado nível da síndrome e a maioria estava se sentindo sobrecarregada, porém satisfeita e não possuía intenção em deixar o emprego. Quanto maior o nível de Burnout e a exaustão emocional maior foram a insatisfação profissional, a intenção em deixar o emprego e o sentimento de sobrecarga. As mulheres mostraram-se mais despersonalizadas e aqueles que já haviam gozado férias estavam mais realizados pessoalmente do que os demais. Avaliar essa síndrome é extremamente importante, pois os resultados de seu desenvolvimento e manifestação podem impactar negativamente nos resultados com os pacientes, profissionais e instituições.

DESCRITORES: Esgotamento profissional; Satisfação no emprego; Enfermagem.

\section{BURNOUT SYNDROME IN THE NURSING TEAM OF A TEACHING HOSPITAL}

\begin{abstract}
This study's objective was to identify the nursing team's level of Burnout Syndrome and its relationship with the variables of work overload, professional satisfaction, and intention to leave the job. Data collection was undertaken in June - August 2011 using Maslach's Burnout Inventory. The sample of 178 professionals demonstrated a moderate level of the syndrome and the majority felt that they were overloaded with work, but were satisfied and did not intend to leave their jobs. The higher the level of Burnout and emotional exhaustion, the higher the professional dissatisfaction, intention to leave the job, and feeling of overload. The women were shown to be more depersonalized and those staff who had had holidays were more personally realized than the others. It is extremely important to evaluate this syndrome, as the results of its developing and being manifested can impact negatively on the results with the patients, professionals and institutions.
\end{abstract}

DESCRIPTORS: Professional exhaustion; Satisfaction at work; Nursing.

\section{SÍNDROME DE BURNOUT EN EL EQUIPO DE ENFERMERÍA DE UN HOSPITAL UNIVERSITARIO}

RESUMEN: El objetivo de este estudio fue identificar el nivel del Síndrome de Burnout en el equipo de enfermería y su relación con las variables sobrecarga de trabajo, satisfacción profesional e intención en salir del empleo. Los datos fueron recogidos entre junio y agosto de 2011, utilizándose el Inventario de Burnout de Maslach. La muestra de 178 profesionales reveló moderado nivel del síndrome y la mayoría se sentía sobrecargada, pero satisfecha y no pretendía salir del empleo. Cuanto mayor el nivel de Burnout y el agotamiento emocional, mayor fueron la insatisfacción profesional, la intención en dejar el empleo y el sentimiento de sobrecarga. Las mujeres se revelaron más despersonalizadas, y quienes ya habían salido de vacaciones estaban más realizados personalmente que los otros. Evaluar ese síndrome es extremamente importante, pues los resultados de su desarrollo y manifestación pueden impactar negativamente los resultados con pacientes, profesionales e instituciones. DESCRIPTORES: Agotamiento profesional; Satisfacción en empleo; Enfermería. 


\section{INTRODUÇÃO}

A necessidade cada vez maior de novas tecnologias, contenção de custos e selos de acreditação traz consigo significativas mudanças no ambiente de trabalho e maiores exigências aos trabalhadores, que, se por um lado acarreta indiscutíveis benefícios à assistência, por outro, sobrecarrega ainda mais a equipe, pois essas exigências contrastam com a falta de pessoas qualificadas, déficit de materiais, baixos salários e precário dimensionamento de pessoal ${ }^{(1-3)}$.

A enfermagem é vista como uma profissão desgastante devido à falta de reconhecimento, recursos, autonomia e baixos salários ${ }^{(3)}$. Autores referem que os salários do enfermeiro brasileiro os obrigam a possuir mais de uma fonte de renda, o que aumenta ainda mais a sobrecarga de trabalho e conseqüentemente, maior desgaste $^{(2)}$.

Além disso, a sobrecarga de trabalho associada às relações pessoais, muitas vezes conflitantes, geram um descontentamento generalizado que acaba afetando os resultados com os pacientes (comprometimento da qualidade da assistência prestada), com as instituições (alta taxa de rotatividade da equipe) e com os profissionais (insatisfação profissional e desenvolvimento da Síndrome de Burnout) ${ }^{(2-3)}$.

Burnout é uma síndrome desenvolvida em resposta às fontes crônicas de estresse, presentes no local de trabalho. É caracterizada por três componentes relacionados, mas independentes: exaustão emocional, despersonalização e diminuição da realização pessoal(4). Esse esgotamento pode ser identificado pelo código Z73.0 na Classificação Internacional de Doenças e, no Brasil, a Previdência Social reconhece a Síndrome como um transtorno mental relacionado ao trabalho ${ }^{(5)}$.

Como sintomas e consequências do Burnout, os indivíduos podem sofrer prejuízos na saúde e no desempenho do trabalho como: ansiedade, depressão ${ }^{(4,6)}$, distúrbios do sono, cefaleia, fadiga crônica, abuso de substâncias ${ }^{(6)}$, aumento da insatisfação com o trabalho, absenteísmo e aumento da rotatividade ${ }^{(3)}$.

A rotatividade, no quadro de trabalhadores de enfermagem de um hospital, representa um problema enfrentado pelos gerentes. A síndrome implica diretamente na qualidade do cuidado e nos custos de desligamento, contratação e treinamento. A perda de profissionais experientes afeta o nível de cuidado prestado ${ }^{(3)}$ e essa situação gera descontentamento e sobrecarga de tarefas para os membros que permanecem no hospital. Uma força de trabalho satisfeita, estável e qualificada, além de sustentar o processo de cuidar, cria também possibilidades efetivas de garantia da qualidade dos serviços prestados ${ }^{(3,7)}$.

A equipe de enfermagem é a que mais tempo passa ao lado do paciente e de seus familiares e por isso, compõe um grupo de grande risco ao desenvolvimento do Burnout. As implicações da síndrome trazem consequências relevantes aos profissionais, que podem se sentir insatisfeitos e sobrecarregados com o trabalho; aos pacientes, que podem sofrer prejuízo na qualidade da assistência e às instituições, que podem ser afetadas por meio das altas taxas de absenteísmo e rotatividade.

Portanto, o objetivo do presente estudo foi identificar o nível de Burnout da equipe de enfermagem de um hospital universitário e sua relação com as variáveis sobrecarga de trabalho, satisfação profissional e intenção em deixar o emprego a fim de auxiliar na implementação de intervenções que previnam o desenvolvimento e a manifestação desta síndrome.

\section{MÉTODO}

Pesquisa descritiva, exploratória e transversal, realizada em um hospital público do interior do estado de São Paulo, que possui recursos da prefeitura e tem por objetivo a prestação de assistência médico-hospitalar aos usuários do Serviço Único de Saúde.

Para o estudo foram abordados enfermeiros, técnicos e auxiliares de enfermagem que atenderam aos seguintes critérios de inclusão: a) prestadores de assistência direta aos pacientes; b) não estarem de férias ou licença.

Foi elaborada uma ficha de caracterização pela pesquisadora com o objetivo de avaliar o perfil pessoal e profissional da amostra onde foram contemplados dados como: categoria profissional, idade, sexo, tempo de experiência na profissão e na instituição e realização de horas extras. Ao final dessa ficha, foram inseridas 
três questões para avaliar a percepção dos profissionais sobre os resultados extraídos de estudos anteriormente realizados ${ }^{(3,8-9)}$.

Na primeira pergunta, que avaliou a satisfação profissional, os participantes responderam à questão: "Como você se sente em relação ao seu trabalho atual?"; na segunda questão, que abordou a intenção de deixar o emprego, os sujeitos responderam "Com que frequência você pensa em deixar este emprego nos próximos 12 meses?"; na última pergunta, que abordou o sentimento de sobrecarga de trabalho, os participantes responderam "Com que frequência você se sente sobrecarregado com o seu emprego?".

Para as três perguntas todas as opções de respostas. Quanto maior a pontuação, maior a satisfação do profissional com o seu trabalho atual; menor a intenção do profissional em abandonar o seu emprego atual, no próximo ano; e menor o sentimento de sobrecarga percebido pelos profissionais.

Além da ficha de caracterização, os participantes receberam o Inventário de Burnout de Maslach (IBM) adaptado e validado para a cultura brasileira. É um instrumento auto aplicável que tem por objetivo mensurar o desgaste físico e emocional do trabalhador por meio da avaliação do seu sentimento em relação ao seu trabalho ${ }^{(10)}$. Este possui 22 itens distribuídos em três dimensões: exaustão emocional (nove itens), diminuição da realização pessoal (oito itens) e despersonalização (cinco itens) onde é solicitado que a pessoa responda com que freqüência vivencia determinadas situações no seu ambiente de trabalho(10).

A exaustão emocional é um componente fundamental, mas não suficiente para se definir Burnout. É a primeira reação causada em reposta à sobrecarga de trabalho, conflito social e estresse devido às exigências de bons resultados, o que pode acarretar como estratégia de enfrentamento, o distanciamento emocional e cognitivo em relação ao trabalho(4).

A despersonalização ocorre como tentativa de se proteger da exaustão e caracteriza-se pelo distanciamento do trabalho e das outras pessoas. Já a diminuição da realização pessoal caracteriza-se pelo sentimento de inadequação pessoal e profissional, isto é, as pessoas perdem a confiança em si mesmas e na capacidade de se destacarem perante a equipe ${ }^{(5)}$.

Os itens são avaliados por uma escala tipo Likert com cinco pontos: um (nunca); dois (raramente); três (algumas vezes); quatro (frequentemente) e cinco (sempre), ou seja, quanto maior a pontuação maior a exaustão emocional e a despersonalização e menor a diminuição da realização pessoal, pois esta escala possui escore inverso(10).

Para classificar os níveis da Síndrome de Burnout em alto, moderado e baixo, devemse obter os escores mínimos e máximos para cada subescala e em seguida, determinar os percentis 33 e 67 da curva. Um nível moderado é representado em escores médios nas três subescalas. Um baixo nível é representado por baixos escores nas subescalas exaustão emocional e despersonalização e altos escores na subescala diminuição da realização pessoal e um alto nível é representado por altos escores nas subescalas exaustão e despersonalização e baixos escores na subescala diminuição da realização pessoal ${ }^{(10)}$.

A coleta de dados foi realizada nos meses de junho a agosto de 2011, por uma aluna do quarto ano de graduação em enfermagem, treinada pela pesquisadora. Julgou-se importante a inclusão de uma aluna no processo de coleta de dados por considerar que a autora do presente estudo exerce um cargo administrativo dentro da instituição onde a pesquisa foi realizada, e isso poderia comprometer as respostas dos participantes.

Os nomes dos participantes foram obtidos por meio da escala de trabalho mensal da equipe de enfermagem. A aluna abordou todos os profissionais, de forma individual, em suas unidades de trabalho e aqueles que atenderam aos critérios de inclusão participaram do estudo. Os participantes receberam a ficha de caracterização da amostra e o IBM. Os instrumentos foram respondidos e em envelopes lacrados entregues à supervisão e posteriormente, à aluna de enfermagem.

Os dados foram tabulados no programa Excel-Windows/XP ${ }^{\circledR}$ e analisados por um profissional estatístico, por meio do SAS versão 9.2. Foram obtidas frequências absolutas e relativas para as variáveis categóricas e medidas 
de posição das variáveis contínuas. Para definir o nível da Síndrome de Burnout, foram calculadas as médias das respostas dos sujeitos em cada subescala.

A associação entre as subescalas e as variáveis estudadas foi avaliada por meio dos testes de Qui-quadrado e exato de Fisher e a consistência interna foi avaliada por meio do coeficiente Alfa de Cronbach. O nível de significância assumido foi de 5\%.

Foi obtida autorização do diretor administrativo da instituição, para a realização da pesquisa, e parecer favorável do Comitê de Ética em Pesquisa (Protocolo 89/2011). O estudo foi conduzido de acordo com os padrões éticos onde os sujeitos receberam informações sobre os objetivos da pesquisa, a não compensação financeira, o anonimato na divulgação dos resultados e a possibilidade de recusa em

Tabela 1- Caracterização pessoal e profissional da amostra. Jundiaí-SP-Brasil,2011

\begin{tabular}{lcc}
\multirow{2}{*}{ Variáveis } & \multicolumn{2}{c}{ Participantes } \\
\cline { 2 - 3 } & $\mathrm{n}$ & $\%$ \\
\hline Sexo & 153 & 85,9 \\
\hline Feminino & 9 & 5 \\
\hline Masculino & 16 & 9,1 \\
\hline Não respondeu & & \\
\hline Estado Civil & 74 & 41,6 \\
\hline Casado & 63 & 35,4 \\
\hline Solteiro & 21 & 11,8 \\
\hline Divorciado/Separado & 2 & 1,1 \\
\hline Viúvo & 11 & 6,2 \\
\hline Outros & 7 & 3,9 \\
\hline Não respondeu & \\
\hline Outro vínculo empregatício & \\
\hline Não & 129 & 72,5 \\
\hline Sim & 42 & 23,6 \\
\hline Não respondeu & 7 & 3,9 \\
\hline Férias nos últimos seis meses & \\
\hline Não & 98 \\
\hline Sim & 80 & 55,1 \\
\hline Não respondeu & 0 & 0 \\
\hline Hora extra nos últimos seis meses \\
\hline Sim & 119 \\
\hline Não & 56 & 66,8 \\
\hline Não respondeu & 3 & 1,7 \\
\hline
\end{tabular}

responder qualquer item do instrumento a qualquer momento, sem que isso lhe acarretasse qualquer prejuízo.

\section{RESULTADOS}

A amostra foi composta por 178 profissionais (80\% do total de profissionais da instituição) e a maioria estava lotada nas unidades terapia intensiva $(29,2 \%)$ e trabalhava no período noturno $(41,6 \%)$. As demais variáveis de caracterização pessoal e profissional estão apresentadas na Tabela 1.

A média de idade dos participantes foi de $33,4$ anos ( $\mathrm{dp} \pm 8,2)$, de tempo de experiência na profissão de 6,6 anos (dp $\pm 6,9)$ e de tempo de trabalho na instituição de 2,9 anos (dp $\pm 2,6)$.

A avaliação da percepção dos profissionais em relação aos resultados no trabalho está apresentada na Tabela 2.

No que se refere ao sentimento de sobrecarga, intenção em deixar o emprego e satisfação com o trabalho, a maioria respondeu que se sente satisfeita ou muito satisfeita com o emprego

Tabela 2 - Percepção dos profissionais em relação aos resultados do trabalho. Jundiaí-SP-Brasil, 2011

\begin{tabular}{lcc}
\multirow{2}{*}{ Variáveis } & \multicolumn{2}{c}{ Participantes } \\
\cline { 2 - 3 } & $\mathrm{n}$ & $\%$ \\
\hline Satisfação Profissional & & \\
\hline Muito insatisfeito & 9 & 5,1 \\
\hline Insatisfeito & 23 & 12,9 \\
\hline Satisfeito & 108 & 60,6 \\
\hline Muito satisfeito & 35 & 19,7 \\
\hline Não respondeu & 3 & 1,7 \\
\hline Intenção de Deixar o Emprego no Próximo Ano \\
\hline Frequentemente & 13 & 7,3 \\
\hline Algumas vezes & 37 & 20,8 \\
\hline Raramente & 39 & 21,9 \\
\hline Nunca & 89 & 50 \\
\hline Não respondeu & 0 & 0 \\
\hline Sentimento de Sobrecarga de Trabalho \\
\hline Frequentemente & 28 & 15,7 \\
\hline Algumas vezes & 63 & 35,4 \\
\hline Raramente & 51 & 28,7 \\
\hline Nunca & 36 & 20,2 \\
\hline Não respondeu & 0 & 0 \\
\hline
\end{tabular}


atual $(80,4 \%)$, raramente ou nunca pensou em deixar o emprego no próximo ano (71,9\%), mas algumas vezes ou frequentemente se sente sobrecarregada (51,1\%).

Ao relacionar as três variáveis de resultados no trabalho, constatou-se que os profissionais mais satisfeitos possuíam menor intenção em deixar o emprego $(p=0,00)$ e sentiam-se menos sobrecarregados $(p=0,00)$ e os participantes que trabalhavam no centro cirúrgico possuíam maior intenção em abandonar seu trabalho $(p=0,02)$.

$\mathrm{Na}$ Tabela 3 estão representadas as médias e desvios padrão das subescalas do IBM.

No presente estudo, os profissionais relataram um moderado nível de exaustão emocional, despersonalização e diminuição da realização pessoal, demonstrando, portanto, um moderado nível da Síndrome de Burnout.

No estudo das variáveis com relação ao Burnout, as associações significativas encontradas foram: quanto maior o nível da síndrome, maior a insatisfação profissional, a intenção em deixar o emprego no próximo ano e o sentimento de sobrecarga de trabalho $(p<0,0001)$.

Ao analisar as subescalas separadamente, também foi encontrado que quanto maior a exaustão emocional, maior a insatisfação profissional, a intenção em deixar o emprego no próximo ano e o sentimento de sobrecarga $(p<0,0001)$.

Na subescala despersonalização, as mulheres $(p=0,02)$ e aqueles que possuíam outro vínculo $(p=0,0012)$ sentiam-se mais despersonalizados. Na subescala diminuição da realização pessoal, os participantes que não tinham gozado férias nos últimos meses relataram menor realização pessoal $(p=0,04)$. O coeficiente de confiabilidade obtido para o total de itens do instrumento foi de 0,84 , considerado satisfatório.

Tabela 3- Média e desvio padrão das subescalas do Inventário de Burnout deMaslach. Jundiaí-SP,Brasil, 2011

\begin{tabular}{lcc}
\hline Subescalas & Média & Desvio padrão \\
\hline Exaustão emocional & 17,7 & \pm 6 \\
\hline Despersonalização & 7,7 & $\pm 2,7$ \\
\hline $\begin{array}{l}\text { D i m i n u i çã o d a } \\
\text { realização pessoal } \\
\text { (escore inverso) }\end{array}$ & 32,5 & $\pm 5,1$ \\
\hline
\end{tabular}

\section{DISCUSSÃO}

A predominância do sexo feminino encontrada no presente estudo também foi encontrada em inúmeros estudos nacionais e internacional ${ }^{(9,11-12)}$ e isso se deve à história e cultura da enfermagem. Com relação à maioria ser casada e não exercer outro vínculo empregatício, os resultados também corroboram com outros estudos ${ }^{(1,9)}$.

A média de idade da amostra aproximase de outras pesquisas, demonstrando que a enfermagem, no Brasil, é uma profissão exercida basicamente por adultos jovens ${ }^{(1,9,11-12)}$. No que se refere ao tempo de experiência e de trabalho na instituição, os resultados encontrados assemelham-se aos de outros autores que afirmam que os profissionais, por exercerem a profissão há menos de dez anos, são considerados novos e inexperientes ${ }^{(9)}$.

A maioria dos participantes realizou hora extra e não tinha gozado férias nos últimos seis meses e talvez por isso, estava se sentindo sobrecarregada. A enfermagem, por ser uma profissão mal remunerada, obriga os profissionais a buscar novas fontes de renda e, dessa forma, acaba por exercer longas jornadas de trabalho ${ }^{(3,13)}$.

Mesmo com a rotatividade da equipe de enfermagem, sempre em busca de melhores salários, a maioria não possuía ou possuía rara intenção em deixar o emprego no próximo ano. Estes dados podem estar associados ao fato da maioria sentir-se satisfeita ou muito satisfeita com o trabalho atual. Essa relação também foi apontada em outros estudos ${ }^{(7,9)}$.

Na presente pesquisa, os participantes lotados no centro cirúrgico referiram maior intenção de deixar o emprego no próximo ano. Essa relação pode ser explicada pelo fato de que profissionais que se sentem sobrecarregados possuem maior intenção em trocar de emprego e num estudo que teve por objetivo avaliar a exaustão de enfermeiros de unidades críticas, os que trabalhavam no centro cirúrgico foram os que se mostraram mais cansados ${ }^{(14)}$.

Ao analisar a Síndrome de Burnout, a exaustão emocional e outras variáveis, percebeu-se que o profissional que desenvolve a síndrome sentese mais sobrecarregado o que pode provocar insatisfação profissional e por consequência intenção em deixar o emprego. Um profissional 
insatisfeito pode comprometer o processo de trabalho e afetar os resultados com os pacientes e instituição ${ }^{(1,7)}$.

No que se refere à subescala despersonalização, a diferença encontrada em relação ao sexo, pode estar associada ao fato das mulheres exercerem papéis de mãe, esposa e profissional e sendo assim, acabam se dedicando mais aos dois primeiros papéis e por isso, distanciam-se emocionalmente da profissão ${ }^{(11)}$.

Os sujeitos que não possuem outro vínculo empregatício julgaram-se mais despersonalizados, ou seja, mais distantes emocionalmente do trabalho e das outras pessoas do que aqueles que possuem mais de um emprego. Esses achados não corroboram com os da literatura onde autores afirmam que a sobrecarga de trabalho é um dos fatores que contribui para o desenvolvimento da síndrome e quando a despersonalização se manifesta, os indivíduos apresentam atitudes negativas com relação às outras pessoas e inclusive, com os pacientes ${ }^{(9)}$.

Num estudo com o intuito de avaliar o Burnout, umas das estratégias mais sugeridas pelos participantes para se prevenir o desenvolvimento da síndrome foi o envolvimento em atividades extra laborais que promovam o bem estar. $\mathrm{O}$ período de férias representa um momento de descanso das atividades laborais e provavelmente por isso, aqueles que ainda não haviam gozado férias estavam se sentindo menos realizados pessoalmente ${ }^{(15)}$.

O sentimento de sobrecarga e moderado nível da Síndrome de Burnout demonstrados pela maioria dos participantes pode estar associado ao fato da maioria também ter realizado hora extra nos últimos meses, o que demonstra uma inadequação do quadro de profissionais da instituição. Por outro lado, os profissionais possuíam alta satisfação profissional e baixa intenção em deixar o emprego. Essa relação contraditória entre sentimento de sobrecarga e satisfação profissional também foi encontrada em outros estudos nacionais $s^{(3,7,16)}$.

Pesquisas podem ser utilizadas para auxiliar na implementação de ações que capacitem os profissionais a reconhecerem os sinais do desenvolvimento e manifestação da síndrome para que, dessa forma, implementem estratégias eficazes no enfrentamento e gerenciamento dos estressores no ambiente de trabalho ${ }^{(17)}$.

\section{CONSIDERAÇÕES FINAIS}

Os resultados obtidos responderam aos objetivos inicialmente propostos e por meio deles, destaca-se a importância de se estudar o nível da Síndrome de Burnout dos profissionais de enfermagem por seu custo social.

As características do ambiente de trabalho que favorecem a prática profissional da enfermagem podem afetar diretamente os resultados com os pacientes (prejuízo da qualidade da assistência oferecida), com os profissionais (insatisfação profissional, desenvolvimento da burnout) e com a instituição (absenteísmo e rotatividade), por isso, para que se disponha de mais subsídios para avaliação, recomenda-se a realização de novos estudos que analisem o nível da Síndrome de Burnout juntamente com o ambiente de trabalho, considerando que já existe disponível na cultura brasileira, instrumento adaptado e validado para este fim.

\section{REFERÊNCIAS}

1. Jodas DA, Haddad MCL. Síndrome de burnout em trabalhadores de enfermagem de um pronto socorro de hospital universitário. Acta Paul. Enferm. 2009;22(2):192-7.

2. Silva DCM, Loureiro MF, Peres RS. Burnout em profissionais de enfermagem no contexto hospitalar. Psicol. hosp. 2008;6(1):39-51.

3. Grazziano ES, Ferraz Bianchi ER. Impacto del estrés ocupacional y burnout en enfermeros. Enferm. glob. 2010;(18):1-20.

4. Milanés ZC, Batista E, Cantillo C, Jaramilo A, Rodelo $\mathrm{D}$, et al. Desgaste profesional y factores de enfermería de servicios de urgencias de Cartagena, Colombia. Aquichan 2010;10(1):43-51.

5. Trindade LL, Lautert L. Síndrome de burnout entre os trabalhadores da estratégia de saúde da família. Rev Esc Enferm USP 2010;44(2):274-9.

6. Maslach C, Leiter MP. Trabalho: fonte de prazer ou desgaste? Guia para vencer o estresse na empresa. Trad. de Mônica Saddy Martins. Campinas: Papirus; 1999.

7. Gasparino RC, Guirardello EB, Aiken LH. Validation of brazilian version of the Nursing Work Index - Revised. J. Clin. Nurs. 2011;20(23-24):3494-501.

8. Bogaert PV, Meulemans H, Clarke S, Vermeyen K, Heyning PV. Hospital nurse practice environment, burnout, job outcomes and quality of care: test of a structural equation model. J Adv Nurs 2009;65(10):217585. 
9. Meneguini F, Paz AA, Lautert L. Fatores ocupacionais associados aos componentes da síndrome de burnout em trabalhadores de enfermagem. Texto Contexto Enferm. 2011;20(2):225-33.

10. Tamayo MR. Relação entre a síndrome do burnout e os valores organizacionais no pessoal de enfermagem de dois hospitais públicos [Dissertação]. Brasília (DF): Universidade de Brasília; 1997.

11. Moreira DS, Magnago RF, Sakae TM, Magajewski FRL. Prevalência da síndrome de burnout em trabalhadores de enfermagem de um hospital de grande porte da Região Sul do Brasil. Cad. Saúde Pública 2009;25(7):1559-68.

12. Rissardo MP, Gasparino RC. Exaustão emocional em enfermeiros de um hospital público. Esc. Anna Nery 2013;17(1):128-32.

13. Trindade LL, Lautert L. Síndrome de burnout entre os trabalhadores da estratégia de saúde da família. Rev Esc Enferm USP 2010;44(2):274-9.

14. Martino MMF, Misko MD. Estados emocionais de enfermeiros no desempenho profissional em unidades críticas. Rev Esc Enferm USP 2004;38(2):161-7.

15. Roque L, Soares L. Burnout numa amostra de psicólogos portugueses da região autónoma da Madeira. Psic., Saúde \& Doenças 2012;13(1):2-14.

16. Aiken LH, Sloane DM, Clarke S, Poghosyan L, Cho E, et al. Importance of work environments on hospital outcomes in nine countries. Int J Qual Health Care 2011;23(4):357-64.

17. Linch GFC, Guido LA, Umann J. Estresse e profissionais da saúde: produção do conhecimento no centro de ensino e pesquisas em enfermagem. Cogitare enferm. 2010;15(3):542-7. 